\title{
ACUTE SKIN FAILURE E LESÃO POR PRESSÃO EM PACIENTE COM COVID-19
}

\author{
Aline de Oliveira Ramalho ${ }^{1,2, *} \mathbb{B}$, Talita dos Santos Rosa' $\mathbb{D}$, \\ Vera Lúcia Conceição de Gouveia Santos ${ }^{10}$, Paula Cristina Nogueira
}

\section{RESUMO}

Objetivo: relatar o caso de um paciente crítico com COVID-19 e mostrar os principais achados relacionados à lesão considerada Acute skin failure (ASF), bem como realizar seu diagnóstico diferencial com lesão por pressão (LP) evitável. Método: estudo observacional do tipo relato de caso, desenvolvido em um hospital de São Paulo, na unidade de terapia intensiva (UTI) exclusiva a pessoas diagnosticadas com COVID-19. Os dados foram coletados de um único paciente, entre os meses de março e setembro de 2020. Resultados: paciente com complicações da COVID-19 evoluiu com lesão de pele, inicialmente definida como LP e posteriormente reclassificada como ASF. Os seguintes achados corroboraram o diagnóstico: ventilação mecânica invasiva prolongada, insuficiências respiratória, renal e cardíaca e sepse de foco respiratório. Além disso, outros fatores agravantes, como o uso de droga vasoativa, instabilidade hemodinâmica com intolerância ao mínimo reposicionamento, jejum prolongado e coagulopatia intravascular disseminada associada à infecção pelo coronavírus. Conclusão: o relato mostra que existem dificuldades para o diagnóstico diferencial entre ASF e LP na prática clínica. Trata-se de conceito novo, sendo fundamental que o profissional de saúde reconheça os principais fatores associados ao aparecimento da ASF, muitos dos quais também estão relacionados ao desenvolvimento das LP, ressaltando a necessidade de análise individualizada dessas lesões, e garantia da implementação de intervenções adequadas para prevenção e tratamento.

DESCRITORES: Lesão por pressão. Infecções por coronavírus. Cuidados de enfermagem. Unidades de terapia intensiva. Estomaterapia.

\section{ACUTE SKIN FAILURE AND PRESSURE INJURY IN THE PATIENT WITH COVID-19}

\begin{abstract}
Objective: report the case of a critical patient with COVID-19 and show the main findings related to the injury considered acute skin failure (ASF), as well as perform his differential diagnosis with preventable pressure injury (PI). Method: observational, longitudinal, case report type study, developed in a hospital in São Paulo, in the intensive care unit (ICU) exclusively for people diagnosed with COVID-19. Data were collected from a single patient between March and September 2020. Results: A patient with complications from COVID-19 developed a skin lesion, initially defined as PI and later reclassified as ASF. The following findings corroborated the diagnosis: prolonged invasive mechanical ventilation, respiratory, renal and cardiac insufficiency and sepsis of respiratory focus. In addition, other aggravating factors, such as the use of vasoactive drugs, hemodynamic instability with intolerance to minimal repositioning, prolonged fasting and disseminated intravascular coagulopathy associated with coronavirus infection.
\end{abstract}

1. Universidade de São Paulo - Escola de Enfermagem - São Paulo (SP), Brasil.

2. Hospital Sírio Libanês - São Paulo (SP), Brasil.

*Autora correspondente: alineo_ramalho@hotmail.com

Editor de Seção: Juliano Teixeira Moraes

Recebido: Dez. 23, 2020 | Aceito: Fev. 08, 2021

Como citar: Ramalho AO; Rosa TS; Santos VLCG; Nogueira PC. Acute skin failure e lesão por pressão no paciente com Covid-19: um relato de caso. ESTIMA, Braz. J. Enterostomal Ther., 2021, 19: e0521. https://doi.org/10.30886/estima.v19.1007_PT 
Conclusion: the report shows that there are difficulties for the differential diagnosis between ASF and PI in clinical practice. This is a new concept, and it is essential that health professionals recognize the main factors associated with the appearance of ASF, many of which are also related to the development of PI, highlighting the need for individualized analysis of these injuries, and ensuring the implementation of interventions for prevention and treatment.

DESCRIPTORS: Pressure injury. Coronavirus infections. Nursing care. Intensive care units. Stomatherapy.

\section{ACUTE SKIN FAILURE Y LESIÓN POR PRESIÓN EN EL PACIENTE CON COVID-19}

\section{RESUMEN}

Objetivo: Informar los principales hallazgos en un paciente con COVID-19 ingresado en una unidad de cuidados intensivos (UCI), que desarrolló una lesión considerada Insuficiencia cutánea aguda (PPA), así como diferenciar la lesión por presión (LP) de la PPA. Método: estudio observacional, longitudinal y descriptivo, del tipo reporte de caso, desarrollado en un hospital de São Paulo, en la UCl exclusivamente para personas diagnosticadas con COVID-19, entre los meses de marzo a septiembre de 2020. La muestra estuvo conformada por un paciente. Resultados: Un paciente con complicaciones por COVID-19, evoluciona con una lesión cutánea, inicialmente definida como LP, sin embargo, reclasificada como ASK. Los hallazgos que corroboran el diagnóstico fueron: Ventilación mecánica invasiva prolongada, insuficiencia respiratoria, renal y cardíaca y sepsis del foco respiratorio. Además, factores agravantes como el uso de fármacos vasoactivos, inestabilidad hemodinámica con intolerancia al reposicionamiento mínimo, ayuno prolongado, história clínica con varias comorbilidades y coagulopatía intravascular diseminada inducida por infección por coronavirus. Conclusión: El informe muestra que existen dificultades para el diagnóstico diferencial entre PPA y PL en la práctica clínica. Es un concepto novedoso, y es fundamental que el profesional de la salud reconozca los principales factores asociados a la aparición de la PPA, muchos de los cuales también están relacionados con el desarrollo de LP, destacando la necesidad de un análisis individualizado de estas lesiones y asegurando la implementación de intervenciones adecuadas, para la prevención y el tratamiento.

DESCRIPTORES: Úlcera por Presión. Infecciones por Coronavirus. Atención de Enfermería. Unidades de Cuidados Intensivos. Estomaterapia.

\section{INTRODUÇÃO}

O novo coronavírus (SARS-COV-2), descoberto em dezembro de 2019, provocou uma pandemia de grande impacto, devido a sua alta capacidade de disseminação através de contato e gotículas. A infecção causada por ele, também chamada de COVID-19, apresenta diferentes manifestações clínicas, que variam de infecção assintomática à síndrome respiratória aguda grave (SRAG), geralmente com prognósticos incertos que podem acarretar desde a internação hospitalar, muitas vezes em unidade de terapia intensiva (UTI), à evolução fatal ${ }^{1}$.

Acredita-se que os principais mecanismos responsáveis pela gravidade nesses pacientes estão relacionados com a liberação exacerbada de mediadores inflamatórios e a desregulação do sistema de coagulação, o que favorece o desenvolvimento da SRAG e predispõe à necessidade de suporte respiratório, além da instabilidade clínica e hemodinâmica².

Em função da gravidade dos pacientes acometidos, do aumento exorbitante de internações hospitalares, aumento na demanda de suporte respiratório invasivo, aumento do tempo de internação hospitalar e a necessidade de múltiplos dispositivos e terapêuticas para a recuperação desses pacientes ${ }^{1,2}$, os serviços de saúde sentiram-se pressionados, os custos aumentaram demasiadamente e houve sobrecarga de trabalho das equipes de saúde, caracterizando um panorama mundial quase caótico.

Tendo em vista a complexidade encontrada nesses pacientes, alguns podem evoluir com insuficiência múltipla de órgãos, sendo comum o aparecimento concomitante de lesões de pele, geralmente em áreas expostas à pressão. Nesse contexto, o 
National Pressure Injury Advisory Panel (NPIAP) posicionou-se especificamente sobre os riscos para o desenvolvimento de lesão por pressão (LP), haja vista, também, o aumento importante de sua ocorrência. O painel reconhece que o perfil do paciente com COVID-19 é diferente do que víamos previamente, chamando atenção a aspectos fisiopatológicos da doença que corroboram o aparecimento de lesões de pele, destacando a coagulopatia sistêmica, o hipercatabolismo e o déficit nutricional, bem como a tendência desses pacientes à instabilidade clínica e hemodinâmica, necessidade de posicionamento em prona e utilização de múltiplos dispositivos de assistência à saúde. A organização também ressalta a existência de problemas institucionais relacionados à sobrecarga de trabalho, à falta de tempo para preparo da mão de obra contratada e às dificuldades para compra e utilização de produtos direcionados à prevenção de LP³ .

A ocorrência de LP está frequentemente relacionada à quebra nos protocolos de cuidados com a pele ou à inexistência de protocolos baseados em evidência científica, refletindo não só a qualidade da assistência de enfermagem como a excelência do sistema de saúde como um todo ${ }^{4}$. Na maioria dos casos as LP são consideradas como evitáveis ${ }^{5,6}$. Embora existam algumas circunstâncias que favorecem o desenvolvimento de LP de maneira inevitável, somente deve-se considerá-la como tal quando ocorrer mesmo após a avaliação correta do paciente em risco e a implementação de todas as intervenções profiláticas baseadas em evidências ${ }^{5-7}$.

Inúmeras terminologias são utilizadas para as ulcerações inevitáveis na pele, tais como úlcera terminal de Kennedy, lesões de Trombley-Brennan, alterações na pele no final da vida (Skin Changes at Lifés End - SCALE), lesão por pressão inevitável, entre outras ${ }^{8,9}$. Em 2017, Levine ${ }^{10}$ sugeriu a utilização da nomenclatura Skin Failure como um termo "guardachuva" para as lesões que decorrem da terminalidade e da gravidade do paciente, com má perfusão tecidual, podendo ser classificada em aguda, crônica ou relacionada à fase final de vida.

A insuficiência cutânea é um conceito unificador definido pela baixa tolerância do tecido e outros fatores, incluindo hipóxia, isquemia, diminuição da entrega de nutrientes e diminuição da depuração de metabólitos tóxicos que podem promover o desenvolvimento de uma lesão de reperfusão ${ }^{10}$.

A falência aguda da pele ou Acute Skin Failure (ASF) não possui uma definição clara na literatura, no entanto, vários autores ${ }^{7,8,10,11}$ se referem como danos na pele decorrentes de processo agudo, em geral, com instabilidade clínica e hemodinâmica, limitando a oferta de nutrientes e oxigênio para esse órgão. Essas lesões geralmente ocorrem devido à hipóxia tecidual, podendo acometer qualquer área do corpo, não necessariamente em proeminência óssea. Como fatores preditores ao seu aparecimento, destacam-se a ventilação mecânica prolongada ( $>72 \mathrm{~h}$ ), a falência múltipla de órgãos ( 2 ou mais sistemas comprometidos), a presença de doença arterial periférica e a sepse ou choque séptico ${ }^{12,13}$ Já a falência crônica da pele acontece em decorrência de doença crônica pré-existente. A falência da pele relacionada à fase final de vida inclui as SCALE e as úlceras terminais de Kennedy ${ }^{7,8,10,14,15}$.

Tendo em vista que as lesões englobadas no conceito de Skin Failure são desenvolvidas mesmo após a implementação de medidas preventivas adequadas ao risco do paciente para LP, é fundamental a realização de uma avaliação global desse evento, considerando não só aspectos clínicos do paciente, mas também tópicos relacionados à instituição hospitalar na qual esse se encontra. Esses tópicos referem-se à existência de protocolos adequados e atualizados para a prevenção de lesões de pele, adesão da equipe às medidas preventivas, anotações adequadas em prontuário e inclusão de justificativas para sua não execução quando indicadas ${ }^{8,14,15,16}$. Além disso, fatores organizacionais também devem ser analisados, tais como recursos materiais e humanos disponíveis, logística adequada para realização das estratégias de prevenção, entre outros.

Apesar dessas e de outras recomendações e posicionamentos, como aqueles recentemente estabelecidos pela NPIAP e alguns dos quais já adotados no país ${ }^{17}$, a literatura disponível ainda não traz evidências científicas que possam associar diretamente a infecção por COVID-19 e o desenvolvimento de lesões de pele decorrentes ou não de pressão ${ }^{3}$.

Conforme mencionado antes, mesmo com todas as recomendações existentes, a prática clínica tem mostrado um número relevante de lesões em áreas de proeminências ósseas, com aspecto isquêmico, similares à LP tissular profunda em pacientes com COVID-19, desenvolvidas durante o uso de ventilação mecânica invasiva, instabilidade hemodinâmica, uso de drogas 
vasoativas, insuficiência múltipla de órgãos, entre outros fatores já conhecidos como preditores para o desenvolvimento de ASF. Nesses casos, o desafio é realizar o diagnóstico diferencial entre LP e ASF.

As ASF ainda são pouco discutidas na literatura, dificultando a sua diferenciação com as LP evitáveis na prática diária. É de extrema importância a realização do diagnóstico diferencial, uma vez que a LP evitável é considerada um evento adverso relacionado à assistência em saúde, passível de impacto jurídico e econômico, e mostra a fragilidade da qualidade institucional, diferentemente das ASF que não compõem os indicadores de qualidade e deveriam ser excluídas do numerador do indicador de $\mathrm{LP}^{8,11,18}$.

Este artigo tem como objetivo relatar o caso de um paciente crítico com COVID-19 e mostrar os principais achados relacionados à lesão considerada ASF, bem como realizar seu diagnóstico diferencial com LP evitável.

\section{MÉTODO}

Trata-se de um estudo observacional, longitudinal, do tipo relato de caso, desenvolvido na UTI exclusiva a pessoas diagnosticadas com COVID-19, em um hospital filantrópico de grande porte localizado no município de São Paulo, entre os meses de março e setembro de 2020. A amostra foi constituída por paciente único, diagnosticado com COVID-19 e ASF, cujo caso foi extraído de um estudo de coorte retrospectivo que avaliou a incidência de LP e desfecho clínico em pessoas acometidas pela COVID-19. O estudo foi aprovado pelo Comitê de Ética e Pesquisa do hospital (Parecer N0 4.462260), com base na resolução 466/2012 do Conselho Nacional de Saúde/Ministério da Saúde. Os pesquisadores receberam o consentimento do familiar responsável pelo paciente, uma vez que ele se encontrava em coma. Foi aplicado o Termo de Consentimento Livre e Esclarecido para a utilização das imagens de evolução da lesão, em duas vias.

\section{RESULTADOS}

A seguir, apresenta-se uma síntese do caso estudado com destaque aos aspectos de diferenciação entre ASF e LP tissular profunda.

\section{Caso Clínico}

A.Z, 76 anos, foi internado em 23 de março de 2020 devido à presença de tosse há 6 dias, febre alta e dispneia, sendo diagnosticada a SRAG relacionada à infecção pelo novo coronavírus. Apresentava os seguintes antecedentes de saúde: hipertensão arterial sistêmica, insuficiência cardíaca obstrutiva, diabetes mellitus, dislipidemia, hipotireoidismo e obesidade. Após dois dias de internação, evoluiu com piora do padrão respiratório, sendo transferido para a UTI e submetido à intubação orotraqueal e ventilação mecânica invasiva, a qual permaneceu até o final da internação. Quatro dias após a hospitalização, apresentou sepse de foco respiratório com necessidade de uso de droga vasoativa (noradrenalina $0,1 \mathrm{mcg} / \mathrm{kg} / \mathrm{min}$, com dose crescente até $0,6 \mathrm{mcg} / \mathrm{kg} / \mathrm{min}$ ), permanecendo em jejum prolongado (> 72h) e anasarca. Evoluiu com insuficiência renal aguda, recebendo tratamento conservador. Em 4 de abril, apresentou coagulação intravascular disseminada (CIVD) e sinais de hipertensão intracraniana, sendo tratado com heparina não fracionada.

Além dessas alterações, nos primeiros dias na UTI apresentou instabilidade hemodinâmica importante, com intolerância ao reposicionamento (dessaturação de oxigênio de até $76 \%$ durante a mobilização). Como solução à mobilização, utilizou-se o recurso eletrônico da cama, que permite lateralização do paciente de maneira gradual e alívio dos pontos de pressão. No entanto, manteve a dessaturação e hipotensão arterial com angulação < 10 graus, tendo que permanecer cerca de 48 horas na mesma posição.

No dia 7 de abril de 2020, detectou-se uma lesão com característica isquêmica em região glútea bilateral, inicialmente definida como LP tissular profunda (Fig. 1). A Tabela 1 apresenta os exames laboratoriais e parâmetros da ventilação mecânica invasiva no decorrer da internação na UTI, antes e após o surgimento da lesão em região glútea. 
Tabela 1. Exames laboratoriais e parâmetros da ventilação mecânica invasiva nos três períodos avaliativos: admissão na unidade de terapia intensiva, antes e após a lesão. São Paulo (SP), Brasil - 2020.

\begin{tabular}{|c|c|c|c|}
\hline Parâmetros hemodinâmicos & $\begin{array}{c}25.03 .2020 \text { - Admissão } \\
\text { na unidade de terapia } \\
\text { intensiva }\end{array}$ & $\begin{array}{l}4.04 .2020-72 \mathrm{~h} \text { antes do } \\
\text { aparecimento da lesão }\end{array}$ & $\begin{array}{c}\text { 7.04.2020 - Identificação da } \\
\text { lesão }\end{array}$ \\
\hline Hemoglobina & $14,4 \mathrm{~g} / \mathrm{dL}$ & $8,2 \mathrm{~g} / \mathrm{dL}$ & $8,6 \mathrm{~g} / \mathrm{Dl}$ \\
\hline Gasometria Arterial & $\begin{array}{c}\mathrm{pH}: 7,40 \\
\mathrm{pO}^{2}: 88 \mathrm{mmHg} \\
\mathrm{pCO}^{2}: 40 \mathrm{mmHg} \\
\mathrm{HCO}^{3}: 24 \mathrm{mmol} / \mathrm{L} \\
\text { BE: } 0,1 \\
\mathrm{SO}^{2}: 97 \%\end{array}$ & $\begin{array}{c}\mathrm{pH}: 7,33 \\
\mathrm{pO}^{2}: 89 \mathrm{mmHg} \\
\mathrm{pCO}^{2}: 62 \mathrm{mmHg} \\
\mathrm{HCO}^{3}: 31 \mathrm{mmol} / \mathrm{L} \\
\text { BE: } 5,2 \\
\mathrm{SO}^{2}: 96 \%\end{array}$ & $\begin{array}{c}\mathrm{pH}: 7,42 \\
\mathrm{pO}^{2}: 79 \mathrm{mmHg} \\
\mathrm{pCO}^{2}: 52 \mathrm{mmHg} \\
\mathrm{HCO}^{2}: 34 \mathrm{mmol} / \mathrm{L} \\
\text { BE: } 8,5 \\
\text { SO }^{2}: 95 \%\end{array}$ \\
\hline Dímero D & 1583 ng/mL FEU & 6522 ng/mL FEU & 5420 ng/mL FEU \\
\hline PCR & 4,14 mg/dL & $4,80 \mathrm{mg} / \mathrm{dL}$ & $8,80 \mathrm{mg} / \mathrm{Dl}$ \\
\hline Creatinina & 1,38 mg/dL & $3,00 \mathrm{mg} / \mathrm{dL}$ & $1,29 \mathrm{mg} / \mathrm{dL}$ \\
\hline Uréia & 56 mg/dL & 272 mg/dL & $151 \mathrm{mg} / \mathrm{dL}$ \\
\hline \multicolumn{4}{|c|}{ Parâmetros da ventilação mecânica invasiva } \\
\hline Parâmetros Respiratórios & $\begin{array}{l}\text { Modo PCV (PC 11; PEEP 10; } \\
\qquad \mathrm{FiO}^{2} \text { 60\%; } 24 / 24 \text { rpm; } \\
\text { TI 0,85/ VC 330mL), } \\
\text { EtCO } 27 \text {, sincrônico a VM. }^{2}\end{array}$ & $\begin{array}{l}\text { Modo VCV (PEEP 12; 30/30 } \\
\text { rpm; TI 0,61; FiO² 45\%; } \\
\text { VC 360ml), EtCO² 43; } \\
\text { sincrônico a VM. }\end{array}$ & $\begin{array}{l}\text { Modo VCV (PEEP 08; } \\
\text { TI 0,58; FiO² 60\%, 24/24 rpm; } \\
\text { VC 400ml; drive pressure 7; } \\
\text { complacência 57; resistência } \\
\text { 7.7; Pressão de pico 25; } \\
\text { Pressão platô 15), EtCO² 40, } \\
\text { sincrônico a VM. }\end{array}$ \\
\hline
\end{tabular}

pH = Potencial de Hidrogênio; $\mathrm{pO}^{2}$ = Pressão Parcial de Oxigênio; PCR = Polymerase Chain Reaction; pCO² = Pressão Parcial de Gás Carbônico; $\mathrm{HCO}^{3}$ = Bicarbonato Básico; $\mathrm{FiO}^{2}$ = Fração Inspirada de Oxigênio; BE = Excesso de Base; SO² = Saturação da Hemoglobina por Oxigênio; FEU = Unidades Equivalentes de Fibrinogênio; PEEP = Pressão Positiva Expiratória Final; VCV = Ventilação com Volume Controlado; PCV/PC = Pressão Controlada; rpm = Respirações por Minuto; TI = Tempo Inspiratório; VC = Volume Corrente; VM = Ventilação Mecânica; EtCO² = Concentração Máxima de Dióxido de Carbono no Final de uma Respiração.

\section{Evolução da lesão}

Após a estabilização clínica, iniciaram-se as abordagens à beira-leito pelo estomaterapeuta. Como conduta inicial, estabeleceu-se um protocolo de observação evolutiva da lesão e proteção da área acometida com espuma de poliuretano multicamadas com silicone e bordas, além da manutenção dos cuidados tópicos, de acordo com as necessidades do paciente, e reforço nas orientações para a equipe, principalmente visando à redistribuição da carga mecânica e consequente redução da pressão na área (Fig. 1 - D1 ao D10).

De acordo com a delimitação da área isquêmica e evolução da área acometida para necrose, foram realizadas aplicações de hidrofibra com prata e hidrogel, visando à absorção vertical do exsudato, gerenciamento da umidade e auxílio no desbridamento autolítico, associados a coberturas de alta tecnologia (espumas de poliuretano com silicone e bordas) (Fig. 1 - D10 ao D40).

Solicitou-se avaliação da equipe de cirurgia plástica que optou por desbridamento parcial da lesão em centro cirúrgico (D40) e posterior abordagem cirúrgica em dois tempos, sendo primeiramente realizado desbridamento e instalação de terapia por pressão negativa (D65) com enfoque na preparação do leito da ferida e fechamento completo da área acometida, no segundo tempo (D100), por meio de retalho miocutâneo de grande extensão (Vide Fig. 1).

Após o tratamento cirúrgico, os cuidados foram direcionados à cicatrização completa da ferida e à proteção da área, mitigando os riscos de desenvolvimento de complicações da ferida operatória e de nova LP. O paciente manteve-se internado na UTI devido à dependência da ventilação mecânica e às sequelas neurológicas. Em agosto, apresentou novo quadro de sepse de foco respiratório, com piora do quadro clínico e hemodinâmico, evoluindo a óbito após 166 dias de internação hospitalar. 


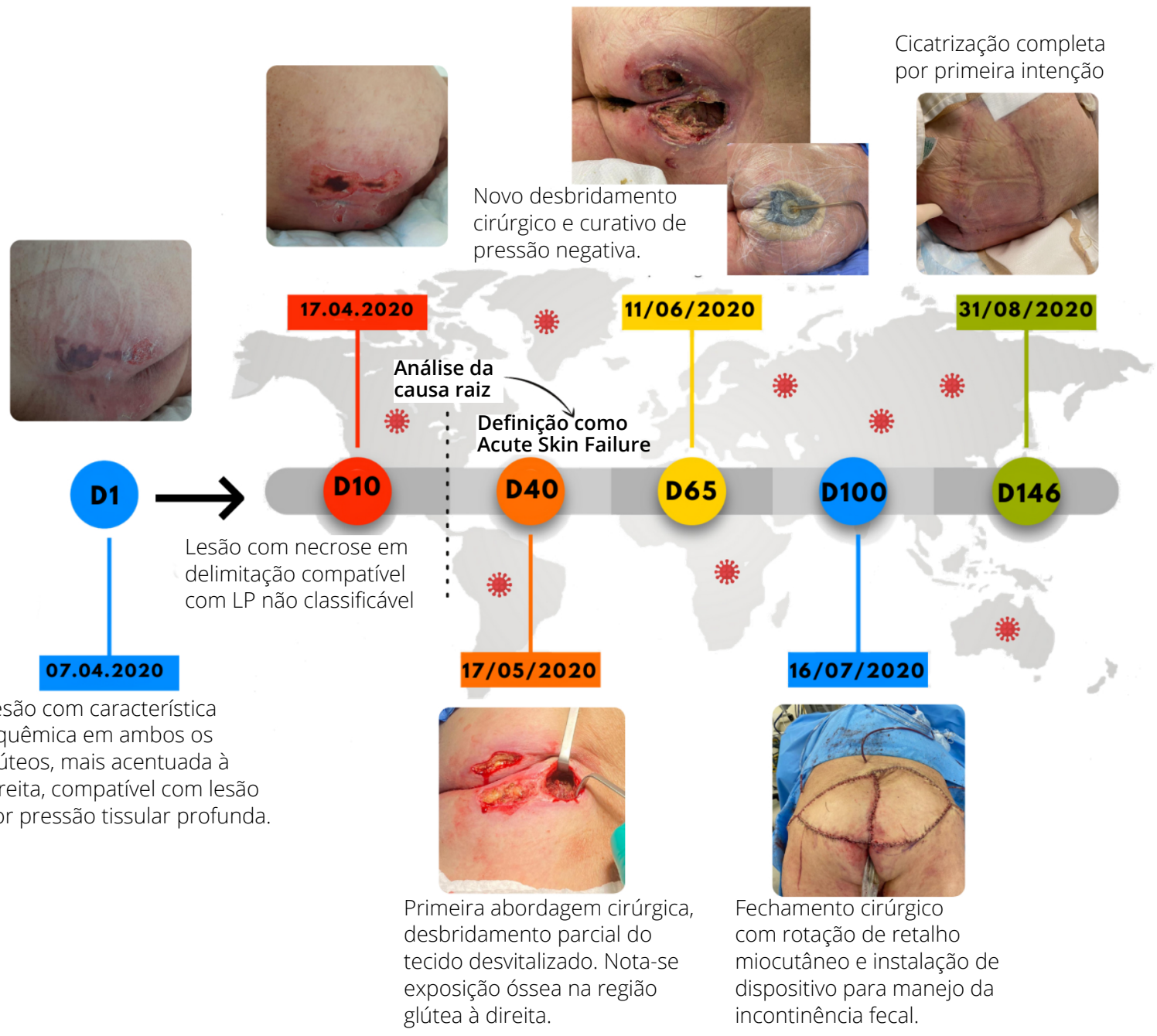

Figura 1. Infográfico da evolução clínica da Acute Skin Failure em decorrência da COVID-19 em paciente internado na unidade de tratamento intensivo. São Paulo (SP), Brasil - 2020.

Conforme rotina institucional, todos os eventos adversos que provocam dano moderado e grave ao paciente devem ser analisados e planos de melhoria do processo implementados, de acordo com cada fragilidade encontrada. Nesse caso, a análise de causa raiz foi conduzida pelos enfermeiros estomaterapeutas da instituição e comitê de integridade da pele (equipe multiprofissional), junto com o núcleo de segurança do paciente.

Nessa análise, identificou-se que as medidas preventivas recomendadas no protocolo institucional foram instituídas, considerando a segurança do paciente e sua tolerância, e justificaram-se, em prontuário, aquelas, pertinentes ao quadro clínico apresentado, que não puderam ser aplicadas.

Considerando, portanto, a presença de instabilidade clínica e hemodinâmica, intolerância mínima ao reposicionamento, coagulopatia sistêmica, déficit de oxigenação decorrente da infecção pelo coronavírus, presença de sepse, tempo prolongado de internação em UTI e ventilação mecânica invasiva, uso de drogas vasoativas, bem como a disfunção multiorgânica (respiratória, cardíaca e renal aguda), pode-se caracterizar e estabelecer a lesão como ASF, excluindo o caso dos indicadores de incidência e prevalência de LP na instituição.

\section{DISCUSSÃO}

Dentro do contexto pandêmico da COVID-19, os serviços de estomaterapia e grupos de prevenção e tratamento de feridas de diversas organizações passaram por modificações e reestruturações, visando a implementar e subsidiar uma 
prática clínica inovadora e abrangente para os pacientes de forma geral. Esses serviços adaptaram-se e atuaram de maneira eficiente mesmo frente a um cenário de incertezas ${ }^{19,20}$. Apesar disso, nota-se um aumento relevante no número de pacientes com lesões de pele, na maioria das vezes, definidas como LP, embora nem sempre constituindo o diagnóstico mais assertivo.

Neste relato de caso, observou-se um grande esforço da equipe multiprofissional para manejo adequado do paciente e para implementação de estratégias de prevenção de LP. Foram aplicadas intervenções de prevenção conforme o protocolo institucional baseado nas recomendações de sociedades internacionais ${ }^{16,21}$, incluindo: avaliação diária do risco de desenvolvimento de LP, utilizando a escala de Braden; inspeção frequente da pele; reposicionamento conforme tolerância do paciente; uso de espumas multicamadas com silicone e bordas como cobertura profilática; superfície de suporte com espuma viscoelástica e alternância de pressão; manejo da umidade; avaliação e intervenção nutricional, entre outras. Mesmo com as intervenções aplicadas, o paciente desenvolveu uma lesão definida inicialmente como LP tissular profunda que acabou sendo diagnosticada definitivamente como ASF, após análise mais detalhada em que foram consideradas, também, as alterações causadas pela COVID-19.

O diagnóstico diferencial de lesões de pele é um desafio, especialmente no ambiente crítico. Por um lado, deve-se ter o diagnóstico diferencial de lesões confundidoras, por outro, há uma luta constante para que a prevenção de LP não seja banalizada e vista como impossível dentro da UTI. Dessa forma, uma vez identificada a LP, a NPIAP recomenda a aplicação da ferramenta de análise de causa raiz para lesões adquiridas no ambiente hospitalar, analisando especialmente o período de $72 \mathrm{~h}$ antes do seu aparecimento ${ }^{22}$. Essa ferramenta pode auxiliar no diagnóstico diferencial e no levantamento de possíveis preditores de lesões consideradas inevitáveis e/ou decorrentes de falência aguda da pele.

A diferenciação entre a LP evitável e a lesão ASF está, portanto, no fator causal e na implementação das medidas preventivas. As LP decorrem especificamente da pressão, fricção e cisalhamento em áreas de proeminências ósseas ou em uso de dispositivos médicos ${ }^{21}$. Já as ASF têm as instabilidades clínica e hemodinâmica como principais fatores causadores, ao resultarem em hipóxia tecidual que acomete áreas distintas do corpo, em proeminências ósseas ou nã $0^{5,7,14}$.

Em documento sobre os aspectos relacionados à complexidade do paciente com COVID-19 e o surgimento de LP inevitáveis publicado pela NPIAP em $2020^{3}$, logo no início da pandemia, encontram-se a fisiopatologia do vírus, a instabilidade do paciente e os fatores de risco intrínsecos e extrínsecos como causais, o que se assemelha àqueles descritos para as ASF, dificultando ainda mais o diagnóstico diferencial entre ambas. Levine ${ }^{8}$ e Ayello et. al ${ }^{10}$ concordam que lesões que se apresentam nesse cenário de criticidade podem ser melhor definidas como Skin Failure.

A diferenciação e o uso do termo ASF em casos relacionados a instabilidade hemodinâmica e criticidade do paciente são importantes, tanto do ponto de vista da clareza de informações ao paciente e família sobre a etiologia da lesão de pele e perspectivas de evolução, quanto do ponto de vista institucional, em função do impacto jurídico e financeiro, da satisfação do paciente e da qualidade da atenção prestada pelo serviço de saúde.

Refletindo sobre a qualidade assistencial, as agências certificadoras que oferecem selos de qualidade do serviço podem dificultar o acesso à certificação caso a diferenciação da etiologia entre LP evitável e ASF não seja precisa. Nesse contexto, as ASF devem ser excluídas do indicador de incidência e prevalência de LP, pois o seu desenvolvimento não está somente relacionado à assistência prestada pela equipe de saúde, mas também à instabilidade clínica e às condições intrínsecas do paciente. Reitera-se que essa exclusão deve ser realizada somente após a análise da execução de estratégias de prevenção de maneira adequada e seguindo as últimas recomendações nacionais e internacionais ${ }^{21,23}$.

No caso relatado, embora os achados de parâmetros da ventilação mecânica e gasometria arterial não tenham mostrado grandes alterações, o paciente apresentou queda acentuada da hemoglobina, aumento gradativo da Polymerase Chain Reaction (PCR) e piora na Creatinina e Dímero D (Tabela 1), anterior ao surgimento da lesão. Além disso, fez uso de vasopressor, permaneceu em jejum prolongado devido à instabilidade, apresentou anasarca e quadro de sepse. Nota-se, também, a piora dos parâmetros ventilatórios (Tabela 1) que implicou em necessidade de bloqueio neuromuscular e houve intolerância ao mínimo reposicionamento.

Embora se disponham de poucas evidências que relacionam a fisiopatologia da infecção por COVID-19 com lesões de pele, sabe-se que a doença compromete a oxigenação e nutrição tecidual bem como favorece o desenvolvimento de coagulopatia disseminada, fatores que podem promover a ocorrência das $\mathrm{ASF}^{3}$. Os dados apresentados no presente estudo de caso, ou seja, quadro de insuficiências respiratória, cardíaca e renal aguda e uso de ventilação mecânica invasiva prolongada, parecem 
corroborar o diagnóstico de ASF ao se compatibilizarem com os fatores descritos como preditores desse desenvolvimento, presentes em revisão sistemática recentemente publicada ${ }^{14}$.

A diferenciação entre as LP evitáveis e as lesões de pele inevitáveis, tais como as ASF, é um processo complexo e demanda uma avaliação detalhada dos achados que fundamentam a tomada de decisão diagnóstica frente às lesões. Após análise detalhada do caso, levantamento da implementação de medidas preventivas proporcionais ao risco do paciente, bem como reflexão da equipe multiprofissional sobre a patologia, quadro clínico e estratégias adotadas para o paciente em questão em conjunto com o time de estomaterapia da instituição, a lesão, anteriormente definida como LP tissular profunda e considerada como um evento adverso, foi redefinida como ASF.

Especialmente no contexto pandêmico em que nos encontramos, estratégias de prevenção de lesões de pele baseadas em evidências são necessárias, associadas à atuação em equipe multiprofissional e a planos de prevenção em condições especiais, como é o caso do posicionamento em prona, prevenção de LP relacionada a dispositivos médicos, dentre outras ${ }^{3,16,17}$. É crucial que o enfermeiro e a equipe de assistência à saúde tenham conhecimentos sobre os fatores preditores de ambos os tipos de lesão, pois a sua diferenciação tem impactos jurídico, econômico e sobre a qualidade assistencial da instituição ${ }^{18}$.

Por se tratar de um tema recente e pouco abordado, há escassez de literatura nessa temática, especialmente no Brasil. Soma-se a isso a carência de instrumentos validados e critérios diagnósticos bem estabelecidos para a diferenciação entre LP e ASF. Nessa perspectiva, embora com limitações pelo tipo de estudo, este relato de caso visa a contribuir para o conhecimento sobre as ASF, seu diagnóstico e diferenciação com as LP, bem como para a reflexão sobre a falência da pele e lesões inevitáveis na prática do cuidado.

\section{CONCLUSÃO}

Espera-se que este relato de caso contribua com algumas respostas às lacunas sobre as Skin Failure na prática clínica, principalmente dos enfermeiros especialistas solicitados para a avaliação e planejamento terapêutico desse tipo de lesão mais complexa. A enfermagem e, particularmente, as especialidades que lidam com o cuidado de pacientes com feridas carecem do desenvolvimento de pesquisas nessa temática, visando a fundamentar critérios e a subsidiar diagnósticos e intervenções mais precisas e eficazes correspondentes aos tipos de lesões, especialmente no paciente crítico com COVID-19.

\section{CONTRIBUIÇÃO DOS AUTORES}

Conceitualização: Ramalho AO e Rosa TS; Metodologia: Rosa TS e Ramalho AO; Redação - Primeira Versão: Ramalho AO e Rosa TS; Redação - Revisão \& Edição: Ramalho AO, Rosa TS, Santos VLCG e Nogueira PC; Supervisão: Santos VLCG e Nogueira PC.

\section{AGRADECIMENTOS}

Ao grupo de estomaterapia do Hospital Sírio Libanês e ao grupo de pesquisa em estomaterapia da Escola de Enfermagem da USP (GPET).

\section{REFERÊNCIAS}

1. Guan W, Ni Z, Hu Y, Liang W, Ou C, He J et al. Clinical Characteristics of Coronavirus Disease 2019 in China. N Engl J Med 2020;382(18):1708-20. https://doi.org/10.1056/NEJMoa2002032

2. Cavayas YA, Noël A, Brunette V, Williamson D, Frenette AJ, Arsenault C et al. Early experience with critically ill patients with COVID-19 in Montreal. Can J Anesth 2020;68:204-13. https://doi.org/10.1007/s12630-020-01816-Z

3. Black J, Cuddigan J, Capasso V, Cox J, Delmore B, Munoz N et al. on behalf of the National Pressure Injury Advisory Panel (2020). Unavoidable Pressure Injury during COVID-19 Crisis: A Position Paper from the National Pressure Injury Advisory Panel. Disponível em: https://cdn.ymaws.com/npiap.com/resource/resmgr/white_papers/Unavoidable_in_COVID_Pandemi.pdf 
4. Ayello EA, Sibbald RG. Pressure Injuries: Nursing-Sensitive Indicator or Team- and Systems-Sensitive Indicator? Adv Skin Wound Care 2019;32(5):199-200. https://doi.org/10.1097/01.ASW.0000557754.10070.88

5. Edsberg LE, Langemo D, Baharestani MM, Posthauer ME, Goldberg M. Unavoidable Pressure Injury: state of the science and consensus outcomes. J Wound Ostomy Continence Nurs 2014;41(4):313-34. https://doi.org/10.1097/WON.0000000000000050

6. Schmitt S, Andries MK, Ashmore PM, Brunette G, Judge K, Bonham PA. WOCN Society Position Paper: Avoidable Versus Unavoidable Pressure Ulcers/Injuries. J Wound Ostomy Continence Nurs 2017;44(5):458-68. https://doi.org/10.1097/ WON.0000000000000361

7. Sibbald RG, Ayello EA. Terminal Ulcers, SCALE, Skin Failure and Unavoidable Pressure Injuries: Results of the 2019 Terminology Survey. Adv Skin Wound Care 2020;33(3):137-45. https://doi.org/10.1097/01.ASW.0000653148.28858.50

8. Ayello EA, Levine JM, Langemo D, Kennedy-Evans KL, Brennan MR, Sibbald RG. Reexamining the Literature on Terminal Ulcers, SCALE, Skin Failure and Unavoidable Pressure Injuries. Adv Skin Wound Care 2019;32(3):109-21. https://doi.org/10.1097/01. ASW.0000553112.55505.5f

9. Langemo DK, Brown G. Skin Fails Too: Acute, Chronic, and End-Stage Skin Failure. Adv Skin Wound Care 2006;19(4):206-11. https://doi.org/10.1097/00129334-200605000-00014

10. Levine JM. Unavoidable Pressure Injuries, Terminal Ulceration, and Skin Failure: In Search of a Unifying Classification System. Adv Skin Wound Care 2017;30(5):200-2. https://doi.org/10.1097/01.ASW.0000515077.61418.44

11. Delmore B, Cox J, Rolnitzky L, Chu A, Stolfi A. Differentiating a pressure ulcer from acute skin failure in the adult critical care patient. Adv Ski Wound Care 2015;28(11):514-24. https://doi.org/10.1097/01.ASW.0000471876.11836.dc

12. Nowicki JL, Mullany D, Spooner A, Nowicki TA, Mckay PM, Corley A et al. Are pressure injuries related to skin failure in critically ill patients? Aust Crit Care 2018;31(5):257-63. https://doi.org/10.1016/j.aucc.2017.07.004

13. Delmore B, Cox J, Smith D, Chu AS, Rolnitzky L. Acute Skin Failure in the Critical Care Patient. Adv Skin Wound Care 2020;33(4):192201. https://doi.org/10.1097/01.ASW.0000604172.69953.23

14. Dalgleish L, Campbell J, Finlayson K, Coyer F. Acute Skin Failure in the Critically Ill Adult Population: A Systematic Review. Adv Skin Wound Care 2020;33(2):76-83. https://doi.org/10.1097/01.ASW.0000617844.69248.92

15. Sibbald RG, Krasner DL, Lutz JB, Sylvia C, Alvarez O, Ayello EA et al. The SCALE Expert Panel: Skin Changes At Life's End. Final Consensus Document. 2009.

16. European Pressure Ulcer Advisory Panel, National Pressure Injury Advisory Panel and Pan Pacific Pressure Injury Alliance. Prevention and Treatment of Pressure Ulcers: Quick Reference Guide. EPUAP, NPIAP, PPPIA: 2016.

17. Ramalho AO, Freitas PSS, Moraes JT, Nogueira PC. Reflexões sobre as recomendações para prevenção de lesões por pressão durante a pandemia de covid-19. ESTIMA, Braz. J. Enterostomal Ther., 2020, 18: e2520. https://doi.org/10.30886/estima. v18.940_PT

18. Kim JH, Shin HK, Jung GY, Lee DL. A case of acute skin failure misdiagnosed as a pressure ulcer, leading to a legal dispute. Arch Plast Surg 2019;46(1):75-8. https://doi.org/10.5999/aps.2018.00087

19. Gray M. Context for Practice: WOC Specialty Practice at the Front Lines during a Global Pandemic. J Wound Ostomy Continence Nurs 2020;47(4):309-10. https://doi.org/10.1097/WON.0000000000000670

20. Pontieri-Lewis V. Adapting WOC Nursing Practice to the COVID-19 Pandemic: A View from Here. J Wound Ostomy Continence Nurs 2020;47(4)321-3. https://doi.org/10.1097/WON.0000000000000667

21. Europen Pressure Ulcer Advisory Panel, National Pressure Injury Advisory Panel and Pan Pacific Alliance. Prevention and Treatment of Pressure Ulcers/Injuries: Quick Reference Guide. Emily Haesler (Ed.). EPUAP/NPIAP/PPPIA:2019.

22. Black JM. Root Cause Analysis for Hospital-Acquired Pressure Injury. J Wound Ostomy Continence Nurs 2019;46(4):298-304. https://doi.org/10.1097/WON.0000000000000546

23. Almeida LV de, Bizutti NS, Draganov PB. Impactos da acreditação Magnet Recognition nos serviços de enfermagem: uma revisão narrativa. Rev Adm Saúde (On-Line) 2019;19(77):e198. https://doi.org/10.23973/ras.77.198 